\title{
The expedite boundary element method
}

\author{
N. A. Dumont \& C. A. Aguilar \\ Department of Civil Engineering, \\ Pontifical Catholic University of Rio de Janeiro, Brazil
}

\begin{abstract}
The present developments combine the variationally-based, hybrid boundary element method with a consistent formulation of the conventional, collocation boundary element method in order to establish a computationally less intensive procedure, although not necessarily less accurate, for large-scale, two-dimensional and three-dimensional problems of potential and elasticity, including timedependent phenomena. Both the double-layer and the single-layer potential matrices, $\mathbf{H}$ and $\mathbf{G}$, whose evaluation usually requires dealing with singular and improper integrals, are obtained in an expedite way that circumvents almost any numerical integration - except for a few regular integrals in the case of $\mathbf{H}$. A few numerical examples are shown to assess the applicability of the method, its computational effort and some convergence issues.
\end{abstract}

Keywords: boundary elements, meshless methods, hybrid boundary elements.

\section{Introduction}

The collocation boundary element method (CBEM), whenever applicable, is a simple, powerful numerical analysis tool [1]. The present contribution is an attempt to show that the CBEM can be still more efficient and powerful - and still easier to implement computationally. (A not lesser contribution is the demonstration that simplicity can be achieved without resorting to exotic concepts such as node displacements from corner points or regularizations.)

Some precursory works have already been published on the subject [9] or are being prepared [11]. However, this is the first attempt to summarize the basic concepts that lead to the expedite boundary element method (EBEM) and to show its main features and possibilities of application in an outline that is meant to be itself expedite. 


\section{Problem formulation}

An elastic body is submitted to body forces $b_{i}$ in the domain $\Omega$ and traction forces $\bar{t}_{i}$ on part $\Gamma_{\sigma}$ of the boundary. Displacements $\bar{u}_{i}$ are known on the complementary part $\Gamma_{u}$ of $\Gamma$. One is looking for an adequate approximation of the stress field that satisfies equilibrium in the domain,

$$
\sigma_{j i, j}+b_{i}=0 \quad \text { in } \Omega
$$

also satisfying the boundary equilibrium and compatibility equations,

$$
\sigma_{j i} n_{j}=\bar{t}_{i} \quad \text { along } \quad \Gamma_{\sigma}, \quad u_{i}=\bar{u}_{i} \quad \text { on } \quad \Gamma_{u}
$$

where $n_{j}$ is the outward unit normal to $\Gamma$. Indices $i, j$, (also $k, l$ ) may assume values 1,2 or 3 , as they refer to the coordinate directions $x, y$ or $z$, respectively, for a general 3D analysis. Summation is indicated by repeated indices. Particularization to $2 \mathrm{D}$ analysis as well as to potential problems is straightforward.

\subsection{Stress and displacement assumptions}

Three independent fields are used in the following developments. The displacement field is explicitly approximated along the boundary by $u_{i}^{d}$, where ()$^{d}$ means displacement assumption, in terms of polynomial functions $u_{i m}$ with compact support and nodal displacement parameters $\mathbf{d}=\left[d_{m}\right] \in \mathbb{R}^{n^{d}}$, for $n^{d}$ displacement degrees of freedom of the discretized model. An independent stress field $\sigma_{i j}^{s}$, where ()$^{s}$ stands for stress assumption, is given in the domain in terms of some particular solution $\sigma_{i j}^{p}$ plus a series of fundamental solutions $\sigma_{i j m}^{*}$ with global support, multiplied by force parameters $\mathbf{p}^{*}=\left[p_{m}^{*}\right] \in \mathbb{R}^{n^{*}}$ applied at the same boundary nodal points $m$ to which the nodal displacements $d_{m}$ are attached $\left(n^{*}=n^{d}\right)$. Displacements $u_{i}^{s}$ are obtained from $\sigma_{i j}^{s}$. Then,

$$
\begin{gathered}
u_{i}^{d}=u_{i m} d_{m} \quad \text { on } \Gamma \text { such that } u_{i}^{d}=\bar{u}_{i} \text { on } \Gamma_{u} \text { and } \\
\sigma_{i j}^{s}=\sigma_{i j m}^{*} p_{m}^{*}+\sigma_{i j}^{p} \quad \text { such that } \sigma_{j i m, j}^{*}=0 \quad \text { and } \sigma_{j i, j}^{p}=b_{i} \text { in } \Omega \\
\Rightarrow \quad u_{i}^{s}=u_{i m}^{*} p_{m}^{*}+u_{i}^{p}+u_{i s}^{r} C_{s m} p_{m}^{*} \text { in } \Omega
\end{gathered}
$$

where $u_{i m}^{*}$ are displacement fundamental solutions corresponding to $\sigma_{i j m}^{*}$. Rigid body motion is included in terms of functions $u_{i s}^{r}$ multiplied by in principle arbitrary constants $C_{s m} \in R^{n^{r} \times n^{*}}$, where $n^{r}$ is the number of rigid body displacements (r.b.d.) of the discretized problem, as dealt with formally in Definition 1, introduced in Section $4[7,10]$. The fundamental solutions $\sigma_{i j m}^{*}$ are used as weight functions in the CBEM. In the variational BEMs and in the EBEM, in particular, they represent domain interpolation functions.

The third independent field is used to approximate traction forces along the boundary by $t_{i}^{t}$, where ()$^{t}$ means traction assumption, as required in the 
conventional boundary element method, given as

$$
t_{i}^{t}=u_{i \ell} t_{\ell} \quad \text { in the CBEM, }
$$

where $u_{i \ell}$ are polynomial interpolation functions with compact support and $\mathbf{t}=$ $\left[t_{\ell}\right] \in \mathbb{R}^{n^{t}}$ are traction-force parameters. The index $i$ refers to the coordinate directions whereas the index $\ell$ refers to any of the $n^{t}$ traction-force degrees of freedom of the problem (thus denoting both location and orientation), for nodes adequately distributed along boundary segments of $\Gamma$. The interpolation functions $u_{i \ell}$ have the same properties of $u_{i n}$, as presented in eqn (3). Equation (6) holds as $\bar{t}_{i}=u_{i \ell} \bar{t}_{\ell}$ along $\Gamma_{\sigma}$, in particular, according to eqn (2). An improved version of eqn (6) is proposed for problems with curved boundaries,

$$
t_{i}^{t}=u_{i \ell} \frac{|J|_{(\text {at } \ell)}}{|J|} t_{\ell} \equiv t_{i \ell} t_{\ell} \quad \text { in the MBEM, }
$$

which leads to the modified boundary element method (MBEM) [8]. In this equation, $|J|_{(a t \ell)}$ is the value of the Jacobian of the global $(x, y, z)$ to natural $(\xi, \eta)$ coordinate transformation at the nodal point $\ell$ and the term $|J|_{(\text {at } \ell)} /|J|$ features a term in the denominator that cancels the Jacobian term of the infinitesimal boundary segment $\mathrm{d} \Gamma=|J| \mathrm{d} \xi \mathrm{d} \eta$ in the numerator of two integral expressions introduced in eqns (10) and (14). This not only improves the capacity of $t_{i}^{t}$ to represent the traction forces along curved boundary segments but also simplifies the numerical integration of the related terms. In the subsequent developments, one refers to the approximation of the traction forces on $\Gamma$ generically as given in eqn (7), $t_{i}^{t}=t_{i \ell} t_{\ell}$, explaining in the text, whenever explicitly required, whether eqn (6) or (7) is meant.

The numbers of degrees of freedom for traction forces $n^{t}$ and displacements $n^{d}$ are not necessarily the same, since one may need more than one traction-force parameter to represent tractions that are not single valued at the boundary surface, generally at nodes where adjacent boundary segments present different outward normals [8]. Then, it results that $n^{t} \geq n^{d}$, as $t_{\ell}$ in eqns (6) and (7) are tractionforce attributes on boundary segments, whereas $u_{i n}$ in eqn (3) are displacement attributes at nodal points. The fact that $n^{t} \geq n^{d}$ leads to some rectangular matrices - the same eqns (14) and (10) of the CBEM, which have been just referred to, plus a third one, introduced in eqn (21).

\subsection{Boundary approximation of the particular solution}

Although neither conceptually nor formally necessary, the following approximation may render all subsequent equations simpler and more elegant [7]. Given a sufficiently refined boundary mesh, the displacements $u_{i}^{p}$ and the traction forces $t_{i}^{p}$ related to an arbitrary particular solution of the non-homogeneous governing eqn (1), whenever available, can be approximated accurately enough by nodal displacement parameters $\mathbf{d}^{p}=\left[d_{n}^{p}\right] \in \mathbb{R}^{n^{d}}$ and traction force parameters $\mathbf{t}^{p}=\left[t_{\ell}^{p}\right] \in \mathbb{R}^{n^{t}}$, respectively, in terms of the interpolation functions of eqns (3) 
182 Boundary Elements and Other Mesh Reduction Methods XXXIII

and (6) or (7):

$$
u_{i}^{p} \approx u_{i n} d_{n}^{p}, \quad t_{i}^{p} \approx t_{i \ell} t_{\ell}^{p} \quad \text { on } \Gamma
$$

One assumes with the above equations that a particular solution for the domain forces $b_{i}$ in eqn (1) is known in terms of displacements $u_{i}^{p}$ and stresses $\sigma_{i j}^{p}$. The means to obtain such particular solutions other than in close form are not discussed herein (see, for instance, Partridge et al [14]).

\section{Conventional and modified boundary element methods}

The matrix equation of the CBEM [1] may be expressed as [8]

$$
\mathbf{H}\left(\mathbf{d}-\mathbf{d}^{p}\right)=\mathbf{G}\left(\mathbf{t}-\mathbf{t}^{p}\right)
$$

where $\mathbf{H}=\left[H_{m n}\right] \in \mathbb{R}^{n^{d} \times n^{d}}$ is a kinematic transformation matrix $[5,7,10]$ and $\mathbf{G}=\left[G_{m \ell}\right] \in \mathbb{R}^{n^{d} \times n^{t}}$ is a flexibility-like matrix (that is in general rectangular, as proposed). The formal definition of these matrices is

$$
H_{m n}=\int_{\Gamma} \sigma_{j i m}^{*} \eta_{j} u_{i n} \mathrm{~d} \Gamma, \quad G_{m \ell}=\int_{\Gamma} t_{i \ell} u_{i m}^{*} \mathrm{~d} \Gamma
$$

The double-layer and single-layer potential matrices $H_{m n}$ and $G_{m \ell}$ comprise in their definition singular and improper integrals, respectively, when source $(m)$ and field (either $n$ or $\ell$ ) indexes refer to the same nodal points. The singular integrals can be always evaluated mathematically in correspondence to simple mechanical meanings [1]. A conceptual assessment of eqn (10) is given in Reference [8].

\section{Some virtual-work statements}

Several virtual-work statements are outlined in the following. They are actually theorems that must be proven from some mechanical axioms. Some of them have already been dealt with in References [6-10]. In this paper, one attempts to keep full consistency of the equations while being not too formal. This compromise is hopefully achieved with the following definition.

Definition 1 Let $n^{r}$ be the number of independent r.b.d. of an elasticity problem, in general. Then, $n^{r}=3$ or 6 for $2 D$ or $3 D$ problems (and $n^{r}=1$ for potential problems) Problems involving symmetry present different values of $n^{r}$. One may eventually have $n^{r}=0$, as for an infinite domain. The r.b.d. $W \in R^{n_{d}}$ are spanned by the columns of a matrix $\mathbf{W} \in R^{n_{d} \times n_{r}}$, which is orthogonal, for convenience.

\subsection{Displacement virtual-work statement}

Part of the Hellinger-Reissner potential $[5,10]$ leads to the equilibrium equation

$$
H_{m n} p_{m}^{*}=p_{n}-p_{n}^{p} \quad \text { or } \quad \mathbf{H}^{\mathrm{T}} \mathbf{p}^{*}=\mathbf{p}-\mathbf{p}^{p}
$$

in which $\mathbf{H}=\left[H_{n m}\right] \in \mathbb{R}^{n^{d} \times n^{*}}$ is the same double layer potential matrix of the collocation boundary element method [1], already introduced in eqn (10). 
Moreover, $\mathbf{p}=\left[p_{n}\right] \in \mathbb{R}^{n^{d}}$ and $\mathbf{p}^{p}=\left[p_{n}^{p}\right] \in \mathbb{R}^{n^{d}}$, defined as

$$
p_{n}=\int_{\Gamma} \sigma_{j i} n_{j} u_{i n} \mathrm{~d} \Gamma, \quad p_{n}^{p}=\int_{\Gamma} \sigma_{j i}^{p} n_{j} u_{i n} \mathrm{~d} \Gamma
$$

are vectors of equivalent nodal forces corresponding respectively to applied boundary tractions, as given in eqn (2) and to the particular solution of eqn (4).

\subsection{Virtual-work relations between the approximate fields given by $\mathrm{d}$ and $\mathrm{t}$}

It may be convenient to express the boundary traction approximations of eqns (6) or (7) in terms of equivalent nodal forces, obtained from the virtual work statement:

$$
\begin{gathered}
\delta d_{m} p_{m}(\mathbf{t})=\delta d_{m} \int_{\Gamma} u_{i m} t_{i \ell} \mathrm{d} \Gamma t_{\ell} \\
\Rightarrow p_{m}(\mathbf{t})=L_{\ell m} t_{\ell} \quad \text { or } \quad \mathbf{p}(\mathbf{t})=\mathbf{L}^{\mathrm{T}} \mathbf{t}
\end{gathered}
$$

where the interpolation functions of eqns (3) and (7) (as for the MBEM) were used, thus defining

$$
\mathbf{L}=\left[L_{\ell m}\right] \in \mathbb{R}^{n^{t} \times n^{d}}=\int_{\Gamma} t_{i \ell} u_{i m} \mathrm{~d} \Gamma
$$

As given in eqn (13), $\mathbf{L}^{\mathrm{T}}$ performs an equilibrium transformation of tractionforce parameters $\mathbf{t}$ to equivalent nodal forces $\mathbf{p}(\mathbf{t})$. The $\operatorname{argument}(\mathbf{t})$ shows that $\mathbf{p}(\mathbf{t})$ is a function of $\mathbf{t}$, thus one of three possible approximations. Observe that, according to Definition $1, \mathbf{W}^{\mathrm{T}}\left(\mathbf{p}(\mathbf{t})-\mathbf{p}^{p}\right)=\mathbf{W}^{\mathrm{T}} \mathbf{L}^{\mathrm{T}}\left(\mathbf{t}-\mathbf{t}^{p}\right)=\mathbf{0}$ for a problem consistently formulated.

One may express the contragradient statement

$$
\mathbf{p}(\mathbf{t})=\mathbf{L}^{\mathrm{T}} \mathbf{t} \quad \Rightarrow \quad \mathbf{d}^{t}(\mathbf{d})=\mathbf{L} \mathbf{d}
$$

where $\mathbf{d}^{t}(\mathbf{d})$ are equivalent nodal displacements defined such that $\delta \mathbf{t}^{\mathrm{T}} \mathbf{d}^{t}(\mathbf{d})$ has the meaning of virtual work. This contragradient statement is part integrand of the hybrid displacement BEM, which may be derived from the Hu potential $[4,7,13]$.

\subsection{Virtual-work relations between the approximate fields given by $\mathrm{d}$ and $\mathrm{p}^{*}$}

One obtains from eqn (11) the contragradient relation

$$
\mathbf{p}\left(\mathbf{p}^{*}\right)=\mathbf{H}^{\mathrm{T}} \mathbf{p}^{*} \quad \Rightarrow \quad \mathbf{d}^{*}(\mathbf{d})=\mathbf{H d}
$$

where $\mathbf{d}^{*}(\mathbf{d})$ are equivalent nodal displacements defined such that $\delta \mathbf{p}^{* \mathrm{~T}} \mathbf{d}^{*}(\mathbf{d})$ has the meaning of virtual work.

\subsection{Subspaces of admissible forces for the field approximations}

The matrix $\mathbf{W}$ of nodal r.b.d. was introduced in Definition 1, which is also the subspace of forces $\mathbf{p}$ that are not in balance. As remarked after eqn (14), the 
columns of $\mathbf{W}^{\mathrm{T}} \mathbf{L}^{\mathrm{T}}$ span the subspace of forces $\mathbf{t}$ that are not in balance [6]. For a finite domain, the columns of $\mathbf{W}$ are the null space of $\mathbf{H}$. Then, one obtains for consistency of eqn (11) that balanced forces $\mathbf{p}^{*}$ must be orthogonal to the null space $\mathbf{V}$ of $\mathbf{H}^{\mathrm{T}}$ [5]. These conclusions are formalized in the following theorem.

Theorem 1 The columns of the matrices $\mathbf{W}, \mathbf{L W}$ and $\mathbf{V}$ span the subspaces of r.b.d. of the approximating fields represented by the parameters $\mathbf{d}, \mathbf{d}^{t}$ and $\mathbf{d}^{*}$, respectively. Each one of the vectors $\mathbf{p}, \mathbf{t}$ and $\mathbf{p}^{*}$ represent nodal forces that are in equilibrium if and only if $\mathbf{W}^{\mathrm{T}} \mathbf{p}=\mathbf{0}, \mathbf{W}^{\mathrm{T}} \mathbf{L}^{\mathrm{T}} \mathbf{t}=\mathbf{0}$ and $\mathbf{V}^{\mathrm{T}} \mathbf{p}^{*}=\mathbf{0}$, respectively.

\subsection{An approximation of the double-layer potential matrix $\mathrm{H}$}

Equation (5) may be applied to the boundary nodes [7, 10], thus asserting that $u_{i}^{d}$ - from eqn (3) - and $u_{i}^{s}$ should coincide along $\Gamma$ :

$$
\mathbf{U}^{*} \mathbf{p}^{*}+\mathbf{W} \mathbf{C} \mathbf{p}^{*}=\left(\mathbf{d}-\mathbf{d}^{p}\right)
$$

where WCp* accounts for an amount of r.b.d. that cannot be transformed between the approximating fields whose parameters are $\mathbf{p}^{*}$ and $\mathbf{d}$. The above equation is a very simple statement, except that there is an embedded amount of r.b.d. and most important - that the terms of $\mathbf{U}^{*}=\left[U_{m n}^{*}\right] \in \mathbb{R}^{n^{d} \times n^{d}}$ for $m$ and $n$ referring to the same node cannot be directly evaluated.

One may assert on the basis of Theorem 1 that, if the set of parameters $\mathbf{p}^{*}$ in eqn (17) corresponds to forces in balance, then $\mathbf{V}^{\mathrm{T}} \mathbf{p}^{*}=\mathbf{0} \Rightarrow \mathbf{W} \mathbf{C} \mathbf{p}^{*}=\mathbf{0}[10]$ and the following contragradient statement holds:

$$
\mathbf{U}^{*} \mathbf{p}^{*}=\mathbf{d}\left(\mathbf{p}^{*}\right) \Rightarrow \mathbf{U}^{* \mathrm{~T}} \mathbf{p}=\mathbf{d}^{*}(\mathbf{p}), \quad \text { provided that } \mathbf{V}^{\mathrm{T}} \mathbf{p}^{*}=\mathbf{0}, \mathbf{W}^{\mathrm{T}} \mathbf{p}=\mathbf{0}
$$

Then, if one uses eqn (13) to define a set of equivalent nodal forces $\mathbf{p}(\mathbf{t})$ and eqn (16) to define a set of equivalent nodal displacements $\mathbf{d}^{*}(\mathbf{d})$, the right-hand side of the above equation becomes

$$
\mathbf{U}^{* \mathrm{~T}} \mathbf{L}^{\mathrm{T}} \mathbf{t}=\mathbf{H d}
$$

By comparing this equation with eqn (9), one concludes that

$$
\mathbf{U}^{* \mathrm{~T}} \mathbf{L}^{\mathrm{T}} \approx \mathbf{G}
$$

which can be formally obtained in the frame of an energy theorem $[10,11]$.

\subsection{An approximation of the single-layer potential matrix $G$}

Equation (17) was obtained by simply asserting that eqn (3) should hold for nodal points along $\Gamma$ (it actually has a variational basis $[5,7,10]$ ). A similar assertion may be made for traction forces along $\Gamma$,

$$
\mathbf{T}^{*} \mathbf{p}^{*}=\mathbf{t}\left(\mathbf{p}^{*}\right)
$$

with the introduction of the matrix $\mathbf{T}^{*}=\left[T_{\ell m}^{*}\right] \in \mathbb{R}^{n^{t} \times n^{*}}$ of traction forces, obtained by measuring the effect $\sigma_{j i m}^{*} n_{j}$ at a boundary node and direction 
characterized by $\ell$ caused by a unit force $p_{m}^{*}$, according to eqn (4). Application of a contragradient statement leads to [11]

$$
\mathbf{T}^{*} \mathbf{p}^{*}=\mathbf{t}\left(\mathbf{p}^{*}\right) \quad \Rightarrow \quad \mathbf{T}^{* T} \mathbf{d}^{t}=\mathbf{d}^{*}\left(\mathbf{d}^{t}\right), \quad \text { r.b.d. excluded }
$$

The expression on the right-hand side may be written in an amenable format if one resorts to the expressions of $\mathbf{d}^{t}$ and $\mathbf{d}^{*}$ in eqns (15) and (16):

$$
\mathbf{T}^{* \mathrm{~T}} \mathbf{L d}=\mathbf{H d}, \quad \text { r.b.d. excluded }
$$

which involves only nodal displacements $\mathbf{d}$. Since $\mathbf{H W}=\mathbf{0}$ for a finite $\Omega$, r.b.d. are automatically excluded in the term on the right-hand side.

Then, one may conclude that

$$
\mathbf{T}^{* \mathrm{~T}} \mathbf{L} \approx \mathbf{H}
$$

provided that the terms of $\mathbf{T}^{*}=T_{\ell m}^{*}$, for $m$ and $\ell$ referring to the same nodal point, are somehow evaluated and that, at least, one makes sure that

$$
\mathbf{T}^{* \mathrm{~T}} \mathbf{L W}=\mathbf{0} \quad \text { for a finite } \Omega
$$

\section{The expedite boundary element method}

Equations (20) and (24) are together the expression of the expedite boundary element method - EBEM,

$$
\mathbf{T}^{* \mathrm{~T}} \mathbf{L}\left(\mathbf{d}-\mathbf{d}^{p}\right)=\mathbf{U}^{* \mathrm{~T}} \mathbf{L}^{\mathrm{T}}\left(\mathbf{t}-\mathbf{t}^{p}\right)
$$

as a reasonable approximation of eqn (9) for the CBEM, here repeated for clarity,

$$
\mathbf{H}\left(\mathbf{d}-\mathbf{d}^{p}\right)=\mathbf{G}\left(\mathbf{t}-\mathbf{t}^{p}\right)
$$

provided that the puzzle of obtaining the still undefined coefficients of $\mathbf{U}^{*}$ and $\mathbf{T}^{*}$ is solved. If one prefers to work in terms of equivalent nodal forces, as in the finite element method, eqn (26) can be alternatively written, according to eq. (13), as

$$
\mathbf{T}^{* \mathrm{~T}} \mathbf{L}\left(\mathbf{d}-\mathbf{d}^{p}\right)=\mathbf{U}^{* \mathrm{~T}}\left(\mathbf{p}-\mathbf{p}^{p}\right)
$$

which is an additional, operational advantage of the proposed EBEM.

\subsection{Evaluation of the undefined coefficients of $\mathrm{T}^{*}$}

The matrix $\mathbf{T}^{*}$ of traction forces introduced in eqn (21) is rectangular. However, one is actually interested in obtaining the undefined values of the square matrix given as the product $\mathbf{T}^{* \mathrm{~T}} \mathbf{L}$ of either eqn (26) or (27).

The matrix $\mathbf{L}$, as defined in eqn (14), has the same numbers of rows and columns as $\mathbf{T}^{*}$, but is banded, with non-zero coefficient $L_{\ell m}$ only if the nodal 
displacement $\delta d_{m}$ and the traction-force attribute $t_{\ell}$ refer to the same boundary segment (element).

Since $\mathbf{L}$ is banded, the undefined coefficients of $\mathbf{T}^{*}$ affect the coefficients $(m, n)$ of the product $\mathbf{T}^{* \mathrm{~T}} \mathbf{L}$ that refer to the same boundary element. One concludes that the coefficients $(m, n)$ of this matrix product, when referring to different boundary elements, can be obtained directly. On the other hand, the number of undefined coefficients of the product $\mathbf{T}^{* \mathrm{~T}} \mathbf{L}$ is larger than the number of undefined coefficients of $\mathbf{T}^{*}$. Since there is a strong singularity affecting the coefficients of $\mathbf{T}^{* \mathrm{~T}} \mathbf{L}$, this must be taken into account, as proposed in the following algorithm [11].

\section{Algorithm for the evaluation of the coefficients of $\mathbf{T}^{* \mathbf{T}} \mathbf{L}$}

1. If the indices $(m, n)$ of $\mathbf{T}^{* \mathrm{~T}} \mathbf{L}$ refer to a boundary segment that is not adjacent to a singularity, then just evaluate the coefficient as the indicated product.

2. If the indices $(m, n)$ refer to a node that is adjacent to a singularity, then replace the coefficient with the corresponding value of $\mathbf{H}$, eqn (10), which requires the evaluation of a regular integral ( $u_{i n}=0$ at the singularity point).

3. If the indices $(m, n)$ refer to a node directly affected by a singularity, evaluate the coefficient by forcing the matrix to be orthogonal to rigid-body displacements. (For an unbounded domain, use the complementary, bounded domain. In case of symmetries, when the number of r.b.d. is not sufficient, additionally apply the problem to a simple analytical solution.)

\subsection{Evaluation of the undefined coefficients of $\mathrm{U}^{*}$}

Once the undefined coefficients of the product $\mathbf{T}^{* \mathrm{~T}} \mathbf{L}$ are evaluated, the best and only way of obtaining the undefined coefficients of $\mathbf{U}^{*}$ is by applying either eqn (26) or (27) to a sufficient number of simple solutions. Let either $\left(\mathbf{D}^{a}, \mathbf{T}^{a}\right)$ or $\left(\mathbf{D}^{a}, \mathbf{P}^{a}\right)$ represent a set with a sufficiently large number of simple analytical solutions of the homogeneous differential equations (1) of the problem that is being modeled, given in terms of displacements and of either surface tractions parameters or equivalent nodal forces. The coefficients about the main diagonal of $\mathbf{U}^{*}$ are obtained in such a way that either

$$
\left|\mathbf{T}^{* \mathrm{~T}} \mathbf{L} \mathbf{D}^{a}-\mathbf{U}^{* \mathrm{~T}} \mathbf{L}^{\mathrm{T}} \mathbf{T}^{a}\right|=\min \quad \text { or } \quad\left|\mathbf{T}^{* \mathrm{~T}} \mathbf{L D}^{a}-\mathbf{U}^{* \mathrm{~T}} \mathbf{P}^{a}\right|=\min
$$

with actually more equations than unknowns, in order to have no direction preferences in the evaluation of the coefficients of $\mathbf{U}^{*}$, which leads to a solution in terms of least squares. For potential problems, the number of constant fluxes is either two or three, for 2D or 3D problems, and just one unknown per node. For general elasticity problems, there are either three or six constant stress states, for $2 \mathrm{D}$ or $3 \mathrm{D}$ problems, and either $2 \times 2$ or $3 \times 3$ unknowns (if symmetry is not enforced). This solution scheme is similar to the one adopted in the HBEM for the evaluation of the undefined coefficients of the flexibility matrix $\mathbf{F}$ [2, 3, 5, 10-13]. 


\section{Some numerical examples}

Figure 1 shows on the left an irregularly shaped domain for which some of the equations and concepts outlined in this paper are assessed numerically in the frame of the solution of the 2D Laplace equation. The figure has corner coordinates $(0,0),(10,20),(20,0),(15,35),(0,20),(17,19),(16,22),(21,24)$ and $(22,20)$. The four curved boundary segments have radii of curvature $20,15,4$ and -4 . The numerical model is implemented in Fortran with double precision. The scheme shown in Figure 1 has a total of 124 nodes [8]. The problem is modeled using linear, quadratic and cubic elements with differently refined meshes, for the total numbers of nodes shown in Table 1. A series of patch tests is run for potential fields applied to the models, according to Table 2, where the potential $\ln r / 2 \pi$ refers to either of the indicated source points $A=(-5,2), B=(10,2)$ or $C=(19.5,20.5)$.

In order to estimate the highest numerical accuracy to be expected in the tests, convergence is first assessed for eqn (9) in the frame of either the CBEM, thus using eqn (6), or of the MBEM, which uses eqn (7) for the interpolation of traction

Table 1: Total numbers of nodal points for the numerical model of Figure 1.

\begin{tabular}{l|cccccccc}
\hline Element type & \multicolumn{8}{|c}{ Total number of nodal points } \\
\hline Linear & 31 & 62 & 124 & 248 & 496 & 992 & 1984 & - \\
Quadratic & - & 62 & 124 & 248 & 496 & 992 & 1984 & 3968 \\
Cubic & - & 93 & 186 & 372 & 774 & 1488 & 2976 \\
\hline
\end{tabular}
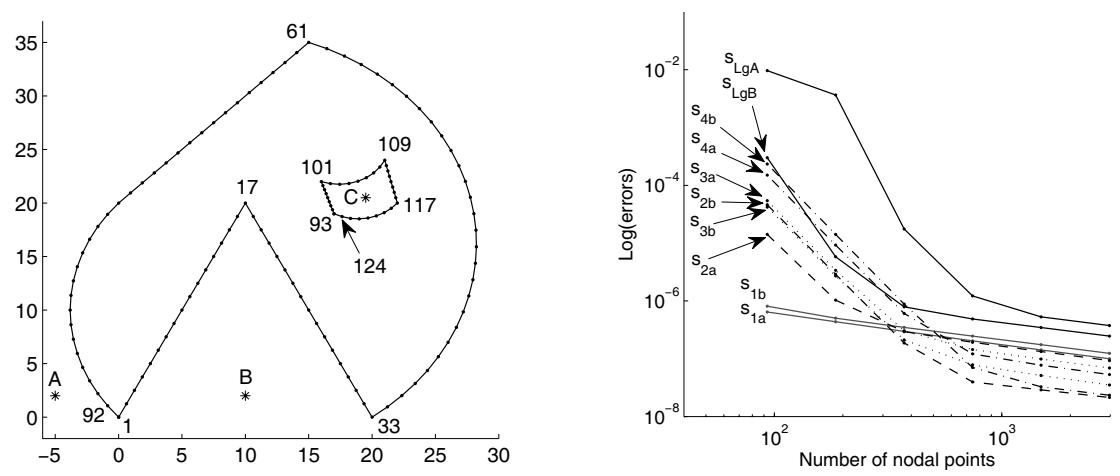

Figure 1: Discretization scheme according to Table 1 and convergence assessments of eqn (9) for the potential fields of Table 2 to establish a benchmark. 
Table 2: Potential fields applied to test the numerical model of Figure 1.

\begin{tabular}{ccccccccc}
\hline$S_{1 a}$ & $S_{1 b}$ & $S_{2 a}$ & $S_{2 b}$ & $S_{3 a}$ & $S_{3 b}$ & $S_{4 a}$ & $S_{4 b}$ & $S_{L g}$ \\
$x$ & $y$ & $x y$ & $x^{2}-y^{2}$ & $x^{3}-3 x y^{2}$ & $y^{3}-3 x^{2} y$ & $x^{4}+y^{4}-6 x^{2} y^{2}$ & $x^{3} y-x y^{3}$ & $\ln r / 2 \pi$ \\
\hline
\end{tabular}

gradients. The results are expressed in terms of the error norm

$$
\epsilon(\mathbf{H d}-\mathbf{G t})=\sqrt{\sum_{m}\left(\sum_{n} H_{m n} d_{n}-\sum_{\ell} G_{m \ell} t_{\ell}\right)^{2} / \sum_{m}\left(\sum_{n} H_{m n} d_{n}\right)^{2}}
$$

for sets of boundary solutions $(\mathbf{d}, \mathbf{t})$ corresponding to the applied potential fields of Table 2. Convergence results for the MBEM with cubic elements are shown on the right of Figure 1. The results for the CBEM are almost indistinguishable from these ones, except when linear potential fields $S_{1 a}$ or $S_{1 b}$ are applied, as in such a case the error norm of eqn (29) is equal to zero (within numerical integration errors) in the MBEM for curved boundaries, which does not happen with the CBEM. Figure 1 shows the expected convergence pattern of a consistently formulated numerical method up to an error norm $\epsilon \approx 10^{-6}$, when numerical integration errors tend to prevail and accuracy hardly improves with increasing mesh refinement.

Figure 2 shows the convergence patterns for the best and worst numerical results obtained for the sets of potential fields of Table 2 . The results on the left correspond to the linear potential field $S_{1 a}$. The CBEM and the MBEM coincide for linear elements $\left(\mathrm{Con}_{l}\right.$ and $\left.\mathrm{Mod}_{l}\right)$, as there are only piecewise straight boundary segments. However, the higher accuracy of the MBEM is flagrant for quadratic and cubic elements $\left(\operatorname{Mod}_{q}\right.$ and $\operatorname{Mod}_{c}$ compared to $\mathrm{Con}_{q}$ and $\left.C o n_{c}\right)$, with exact values given the threshold of numerical integration errors. The corresponding results with
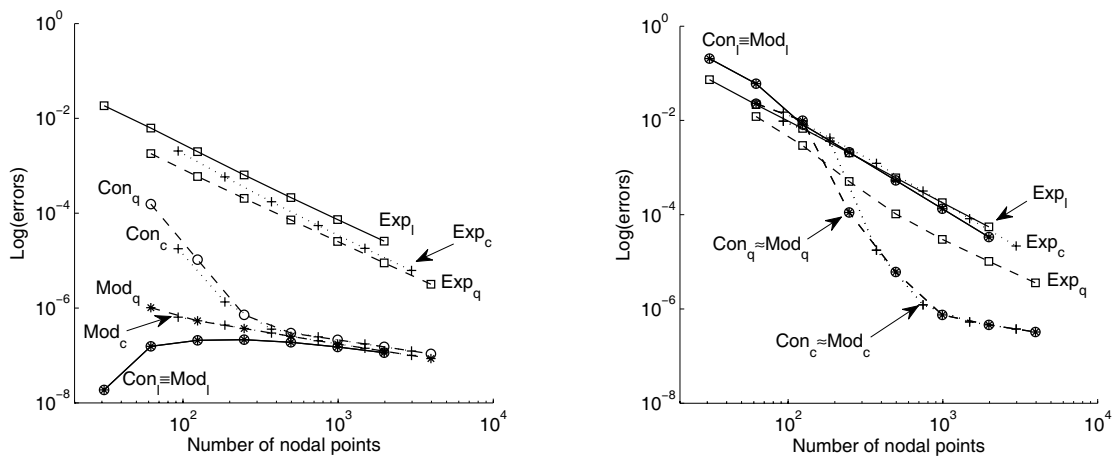

Figure 2: Error norm of eqn (29) for the linear potential field $S_{1 a}$ (on the left) and for a logarithmic field with source at point $C$ in Figure 1. 
the EBEM for linear, quadratic and cubic elements $\left(\operatorname{Exp}_{l}, \operatorname{Exp}_{q}\right.$ and $\operatorname{Exp}_{c}$ are also shown. These graphics actually show the error residuals of the least-square procedure used to evaluate the diagonal coefficients of $\mathbf{U}^{*}$ in eqn (28). Accuracy improves monotonically, although not in the same rate of the CBEM.

The graphics on the right of Figure 2 correspond to the same type of analysis on the left, but for a logarithmic source centered at point $C$ of Figure 1, with error results of eqn (29) that are almost indistinguishable from the ones for a source at point $A$. For high gradient fields, the results with the CBEM and the MBEM are almost the same [8]. The results with the EBEM are initially comparable to the ones of the CBEM. (In the example shown, the results with the EBEM for a coarse mesh are actually better, but no general conclusions can be drawn.) However, the convergence rate is smaller for the EBEM than in the case of the CBEM. The best results with the EBEM, in this and in other examples for $2 \mathrm{D}$ potential problems, are obtained in the implementation with quadratic elements. The results of the EBEM are consistently more accurate than in the implementations of the CBEM using linear elements, a pattern that is also observed in other numerical examples.

\section{Conclusions}

An expedite formulation of the boundary element method is proposed. No integrations are required, except for a few regular ones for a narrow band of coefficients above and below the main diagonal of the matrix that approximates the double-layer potential matrix $\mathbf{H}$. The improved treatment of boundary traction forces leads to simplifications of the conventional BEM itself and to the construction of the auxiliary kinematic/equilibrium transformation matrix $\mathbf{L}$ in a way that also circumvents integration. Although not shown, one may combine the formulation with an efficient technique (GMRES) for the iterative solution of very large equation systems, which also enables the evaluation of results at internal points with no further post-processing. As proposed, the EBEM promises to be superior to the fast multi-pole methods in concept, implementation and computational efficiency. Application of the formulation to time-dependent problems in the frequency domain is straightforward. An extended version of the present manuscript is being prepared, in which numerical examples of threedimensional problems are also shown.

\section{Acknowledgements}

This project was supported by the Brazilian agencies CAPES, CNPq and FAPERJ.

\section{References}

[1] C. A. Brebbia, J. F. C. Telles, and L. C. Wrobel. Boundary Element Techniques. Springer-Verlag, Berlin, 1984. 
[2] R. A. P. Chaves. The Simplified Hybrid Boundary Element Method Applied to Time-Dependend Problems (in Portuguese). PhD thesis, Pontifical Catholic University of Rio de Janeiro, 2003.

[3] M. F. F. de Oliveira and N. A. Dumont. Conceptual completion of the simplified hybrid boundary element method. In E. J. Sapountzakis and M. H. Aliabadi, editors, BETeq 2009 - International Conference on Boundary Element Techniques, pages 49-54, Athens, Greece, July 2009.

[4] T. B. B. DeFigueiredo. A New Boundary Element Formulation in Engineering. Lecture Notes in Engineering. Springer-Verlag, Berlin, 1991.

[5] N. A. Dumont. The hybrid boundary element method: an alliance between mechanical consistency and simplicity. Applied Mechanics Reviews, 42(11):S54-S63, 1989.

[6] N. A. Dumont. An assessment of the spectral properties of the matrix G used in the boundary element methods. Computational Mechanics, 22:3241, 1998.

[7] N. A. Dumont. Variationally-based, hybrid boundary element methods. Computer Assisted Mechanics and Engineering Sciences (CAMES), 10:407430, 2003.

[8] N. A. Dumont. The boundary element method revisited. In C. A. Brebbia, editor, Boundary Elements and Other Mesh Reduction Methods XXXII, pages 227-238. WITPress, Southampton, U.K., 2010.

[9] N. A. Dumont. From the collocation boundary element method to a meshless formulation. In M. A. Storti E. N. Dvorkin, M. B. Goldschmit, editor, Mecánica Computacional, MECOM 2010 - IX Argentinean congress on Computational Mechanics and II South American congress on Computational Mechanics, XXXI CILAMCE - XXXI Iberian Latin-American Congress on Computational Methods in Engineering, pages 4635-4659 (on CD), Buenos Aires, Argentina, 2010.

[10] N. A. Dumont. The hybrid boundary element method - fundamentals (to be submitted). Engineering Analysis with Boundary Elements, 2011.

[11] N. A. Dumont. Toward a meshless formulation of the simplified hybrid boundary element method (to be submitted). 2011.

[12] N. A. Dumont and R. A. P. Chaves. General time-dependent analysis with the frequency-domain hybrid boundary element method. Computer Assisted Mechanics and Engineering Sciences, (10):431-452, 2003.

[13] M. F. F. Oliveira. Conventional, hybrid and simplified boundary element methods (in Portuguese). Master's thesis, Pontifical Catholic University of Rio de Janeiro, 2004.

[14] P. W. Partridge, C. A. Brebbia, and L. C. Wrobel. The Dual Reciprocity Boundary Element Method. Computational Mechanics Publications, Southampton, 1992. 